\title{
Human Anti-Lipopolysaccharid (LPS) antibodies against Legionella with high species specificity
}

\author{
Philipp Kuhn ${ }^{\mathrm{a}, \mathrm{g}}$, Stefanie Thiem ${ }^{\mathrm{b}, \mathrm{c}}$, Michael Steinert ${ }^{\mathrm{b}, \mathrm{d}}$, Duncan Purvis ${ }^{\mathrm{e}}$, Veronika Lugmayr ${ }^{\mathrm{f}}$, \\ Ulrich Treutlein ${ }^{\mathrm{f}}$, Lutz Plobner ${ }^{\mathrm{f}}$, Robert-Matthias Leiser ${ }^{\mathrm{f}}$, Michael Hust ${ }^{\mathrm{a}}$ and Stefan Dübel ${ }^{\mathrm{a}, *}$ \\ ${ }^{a}$ Biotechnology and Bioinformatics, Institute for Biochemistry, Technische Universität Braunschweig, \\ Braunschweig, Germany \\ ${ }^{\mathrm{b}}$ Institute for Microbiology, Technische Universität Braunschweig, Braunschweig, Germany \\ ${ }^{\mathrm{c}}$ Department of Animal Breeding and Genetics, University of Veterinary Medicine Vienna, Vienna, Austria \\ ${ }^{\mathrm{d}}$ Helmholtz Center for Infection Research, Braunschweig, Germany \\ ${ }^{\mathrm{e}}$ Vantix ${ }^{\circledR}$ DIAGNOSTICS, Lowell, MA, USA \\ ${ }^{\mathrm{f}}$ Agrobiogen GmbH, Hilgertshausen, Germany \\ ${ }^{\mathrm{g}}$ YUMAB GmbH, Rebenring, Braunschweig, Germany
}

\begin{abstract}
Legionella are Gram-negative bacteria that are ubiquitously present in natural and man-made water reservoirs. When humans inhale aerosolized water contaminated with Legionella, alveolar macrophages can be infected, which may lead to a lifethreatening pneumonia called Legionnaires' disease. Due to the universal distribution of Legionella in water and their potential threat to human health, the Legionella concentration in water for human use must be strictly monitored, which is difficult since the standard detection still relies on lengthy cultivation and analysis of bacterial morphology. In this study, an antibody against L. pneumophila has been generated from the naïve human HAL antibody libraries by phage-display for the first time. The panning was performed on whole bacterial cells in order to select antibodies that bind specifically to the cell surface of untreated Legionella. The bacterial cell wall component lipopolysaccharide (LPS) was identified as the target structure. Specific binding to the important pathogenic L. pneumophila strains Corby, Philadelphia-1 and Knoxville was observed, while no binding was detected to seven members of the families Enterobacteriaceae, Pseudomonadaceae or Clostridiaceae. Production of this antibody in the recombinant $\mathrm{scFv}-\mathrm{Fc}$ format using either a murine or a human $\mathrm{Fc}$ part allowed the set-up of a sandwich-ELISA for detection of Legionella cells. The scFv-Fc construct proved to be very stable, even when stored for several weeks at elevated temperatures. A sensitivity limit of 4,000 cells was achieved. The scFv-Fc antibody pair was integrated on a biosensor, demonstrating the specific and fast detection of L. pneumophila on a portable device. With this system, 10,000 Legionella cells were detected within $35 \mathrm{~min}$. Combined with a water filtration/concentration system, this antibody may be developed into a promising reagent for rapid on-site Legionella monitoring.
\end{abstract}

Keywords: Phage display, panning, antibody engineering, antibody fragments, Legionella, LPS, biosensor, diagnostics

\footnotetext{
Corresponding author: Stefan Dübel, Technische Universität Braunschweig, Institute for Biochemistry, Biotechnology and Bioinformatics, Spielmannstr. 7, 38106 Braunschweig, Germany. E-mail: s.duebel@tu-bs.de.
}

\section{Introduction}

The first outbreak of the Legionnaires' disease was recorded in 1976. During a convention of the American Legion at the Bellevue Stratford Hotel in Philadelphia, 182 people contracted the disease. 147 of the victims had to be hospitalized and 29 died. The patients 
suffered from a severe pneumonia, accompanied by fever, chills, coughs, headaches or diarrhea [15]. The causative agent was named after the clinical symptoms of the first victims - Legionella pneumophila [7]. Legionella are flagellated Gram-negative bacteria that belong to the class of gamma-proteobacteria. They are ubiquitously present in water habitats, including natural and man-made reservoirs [14,37] where they infect and parasitize protozoa $[1,45]$. In the host, a specialized compartment is formed, that enables replication of Legionella [6].

Humans are infected with Legionella by inhalation of aerosols [13]. Thus, outbreaks are linked to aerosol producing devices like air conditioners [20], showers [46], spa pools [11] or cooling towers [2]. Once having entered the human lung, alveolar macrophages are infected by Legionella, using the same infection strategies as in protozoa [17]. This can lead to a local inflammation and onset of the Legionnaires' disease. At least 63 species or subspecies of the genus Legionella were identified so far. Among them, the serogroup 1 of $L$. pneumophila is the most prevalent pathogen with $84 \%$ of all clinical cases [49].

Since 2001, the Robert Koch-Institute has been registering an increasing number of Legionella infections in Germany. In 2013, approximately 1,000 cases were reported with a lethality rate of 5\% [44]. But it is expected that at least 15,000 to 30,000 infections occur each year that are not recognized by clinicians due to the unspecific symptoms and lack of routine testing in hospitals [47].

Due to the ubiquitous distribution of Legionella in man-made water systems and their potential threat to human health, the German government decided in 2001 to monitor the Legionella levels in drinking water (TrinkwV 2001). If the Legionella concentration exceeds $1 \mathrm{cfu} / \mathrm{mL}$ it is regarded hazardous, demanding disinfection and reporting to health authorities.

Up to now, the standard method for the detection of Legionella in water is microbiological cultivation followed by analysis of microbial morphology. However, this method is time-consuming [5], requires trained laboratory stuff and harbors the risk of under-/over estimation of Legionella if the sample is not treated properly [33]. Therefore, there is a need for a fast, sensitive, and easy manageable diagnostic point-of-care test. These requirements can be met by antibody-based immunosensors as demonstrated for other pathogens [32,35].

In this work, recombinant monoclonal antibodies were selected from the naive human phage display li- braries HAL9/10 [30] by panning on whole L. pneumophila cells. Sandwich pairs were designed and used in portable immunosensor for the detection of $L e$ gionella.

\section{Materials and methods}

\subsection{Preparation of cells for antibody selection}

After washing of a $P$. aeruginosa (NCTC 10662) culture with PBS, cells were inactivated for $2.5 \mathrm{~h}$ at room temperature by formaldehyde treatment $(0.1 \%$ v/v). The L. pneumophila (str. Corby) [24] culture was washed with PBS and directly used for panning.

\subsection{Antibody selection}

Phage $\left(\sim 1 \times 10^{11} \mathrm{cfu}\right)$ of the HAL9/10 libraries [30] were incubated with the formaldehyde fixed $P$. aeruginosa cells $\left(\sim 1 \times 10^{9}\right.$ cells $)$ for $1 \mathrm{~h}$ at room temperature in suspension. Cell bound phage were removed by centrifugation $(10,000 \mathrm{~g}, 5 \mathrm{~min})$, the supernatant was collected and cleared from the remaining cells by filtration $(0.2 \mu \mathrm{m})$. Living Legionella cells $\left(\sim 1 \times 10^{9}\right.$ cells $)$ were diluted in BSA solution $(2 \%)$ and added to the supernatant containing the preabsorbed phage library. After incubation for $1 \mathrm{~h}$ at room temperature, weakly bound or unbound phage were removed by washing with PBS-T $(0.05 \%)$. Legionella bound phage were rescued by infection of $E$. coli, amplified and used for another panning round [16]. In total, three panning rounds were performed. At the end of the third round, $E$. coli cells were infected with selected phage and plated to select single clones as described before [16].

\subsection{Screening-ELISA}

The cavities of a 96-well microtiter plate were filled with 2 YT-GA (150 $\mu \mathrm{L} /$ well) and inoculated with single colonies of the third panning round. After propagation of cells, scFv expression was induced by adjusting to $50 \mu \mathrm{M}$ IPTG for $16 \mathrm{~h}$ at $30^{\circ} \mathrm{C}$. After clearance by centrifugation, the $\mathrm{scFv}$ containing supernatant was screened for cell binding by ELISA.

For this purpose, L. pneumophila (str. Corby) and $P$. aeruginosa cells were diluted in sodium carbonate buffer ( $\mathrm{pH} 9.7$ ) and immobilized for $16 \mathrm{~h}$ at $4^{\circ} \mathrm{C}$ to the cavities of ELISA-plates $\left(4 \times 10^{7}\right.$ cells/well). After blocking and washing with PBS, scFv supernatants were added. Bound $\mathrm{scFv}$ were detected with the Myc1-9E10 antibody in combination with a secondary HRP-conjugated antibody, and TMB as substrate measured at $450 \mathrm{~nm}$. 


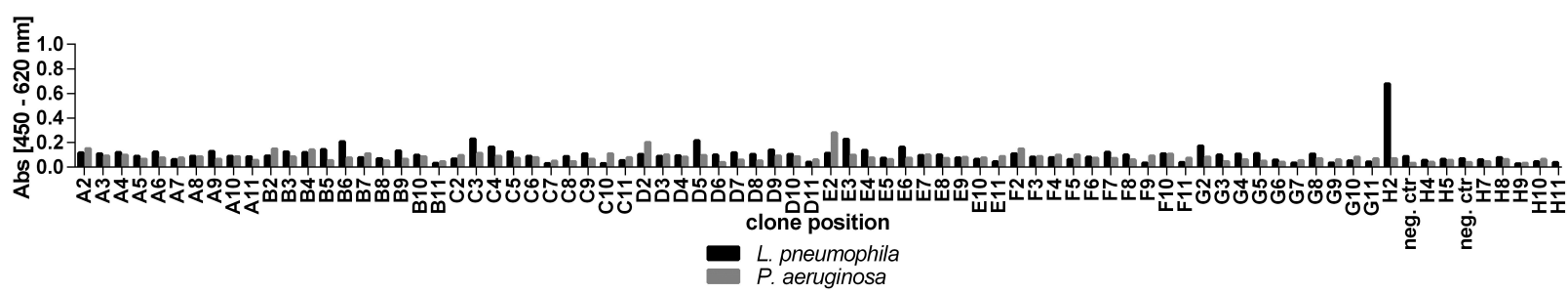

Fig. 1. Identification of monoclonal human $\mathrm{scFv}$ antibodies to L. pneumophila after panning. Whole cell ELISA on immobilized L. pneumophila and textitP. aeruginosa cells of clones enriched by panning of HAL9/10. Shown are the results of 80 clones picked after the first panning campaign. Similar results were obtained for the other pannings. Neg. ctr.: cultivation medium.

\subsection{Production of $s c F v-F c$}

The DNA encoding the $\mathrm{scFv}$ was subcloned in two eukaryotic scFv-Fc expression vectors (pCSE2.6), containing either a human or murine $\mathrm{Fc}$ part, as described before [23]. For production of $\mathrm{scFv}-\mathrm{Fc}$, HEK293-6E cells were transiently transfected. Supernatant was harvested after 7 days and antibodies were purified by Protein A affinity chromatography. The protein concentration was determined by UV/VIS spectrometry and purity was determined by SDSPAGE and Coomassie staining of samples. Binding of the antibodies was tested to non-, heat-, formaldehydeand freeze/thaw treated L. pneumophila, P. aeruginosa and $E$. coli cells by ELISA as described above, but using human-Fc or mouse-Fc specific secondary antibodies for detection.

\subsection{Target identification}

Whole cell lysate was prepared by incubation of Legionella $\left(\sim 1 \times 10^{9}\right.$ cells $)$ for $20 \mathrm{~min}$ at $95^{\circ} \mathrm{C}$ in Laemmli buffer $(200 \mu \mathrm{L})$. For preparation of a protein reduced lysate, Legionella $\left(\sim 1 \times 10^{9}\right.$ cells $)$ were first incubated for $15 \mathrm{~min}$ at $95^{\circ} \mathrm{C}$ in Urea (4 M). Then, Proteinase K $(20 \mu \mathrm{g})$ was added and incubated for $2 \mathrm{~h}$ at $37^{\circ} \mathrm{C}$. The phenol-water extract was prepared as described before [12]. The cell preparations were separated by SDS-PAGE and either Coomassie stained or blotted on a PVDF membrane. After blocking of the membrane, $20 \mu \mathrm{g} \mathrm{scFv-Fc}$ (PHK121-H2h) was added. Bound $\mathrm{scFv}-\mathrm{Fc}$ were detected with a secondary AP-conjugated antisera that catalyzed NBT/BCIP substrate reaction.

\subsection{Detection of L. pneumophila by sandwich-ELISA}

The murine scFv-Fc variant was immobilized to the cavities of ELISA-plates (400 ng/well) for $1 \mathrm{~h}$ at room temperature. After blocking of wells with BSA (1\%) and washing with PBS-T $(0.05 \%)$, various concentrations of heat-treated L. pneumophila (str. Corby) and
E. coli cells were added. After washing with PBS-T $(0.05 \%)$, the captured cells were detected with the human scFv-Fc variant (400 ng/well) as described above. For specificity assays, whole cell lysates of Legionella and various Gram-negative bacterial strains were prepared or cells were heat-treated for $95^{\circ} \mathrm{C}$ for $30 \mathrm{~min}$ and compared by sandwich-ELISA and immunoblot as described above.

\subsection{Stability measurement of PHK121-H2 for long term storage}

The PHK121-H2h antibody was diluted to the final assay concentration in BSA (1\%). Then, aliquots were prepared and stored for different time intervals at $4{ }^{\circ} \mathrm{C}$, RT or $37^{\circ} \mathrm{C}$. Activity of the antibodies was measured by ELISA binding to whole L. pneumophila cells (str. Corby) as described before.

\subsection{Immunosensor measurement}

Electrochemical VR2 sensors (Vantix) were used according to the providers recommendations. In brief, the murine scFv-Fc variant (150 ng) was immobilized on the sensors for $15 \mathrm{~min}$ at $37^{\circ} \mathrm{C}$ in a humidity chamber. After washing, sensors were coated with StabilizingFilm, dried for $2 \mathrm{~h}$ at $37^{\circ} \mathrm{C}$ and stored afterwards at $4^{\circ} \mathrm{C}$. Then, sensors were activated in BSA $(1 \%)$ before a dilution series of heat-treated L. pneumophila (str. Corby) or E. coli cells were added on the sensor. The captured cells were detected with the human scFv-Fc variant $(0.5 \mu \mathrm{g} / \mathrm{ml})$ in combination with a secondary HRP-conjuagted antibody. The sensors were then transferred into Reading-Solution and the HRP activity on the sensors was recorded online.

\section{Results}

\subsection{Human antibody generation by phage display}

To isolate Legionella specific antibodies, three in- 


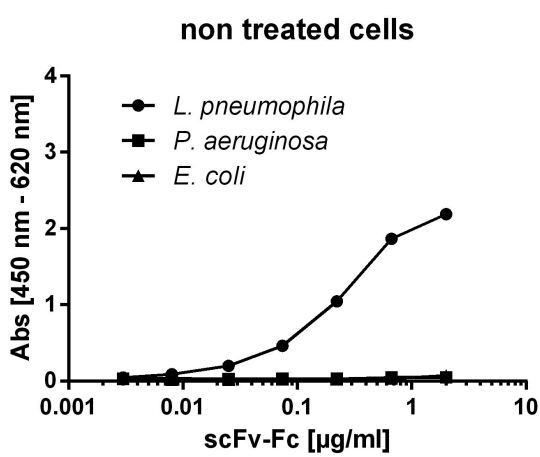

(a)

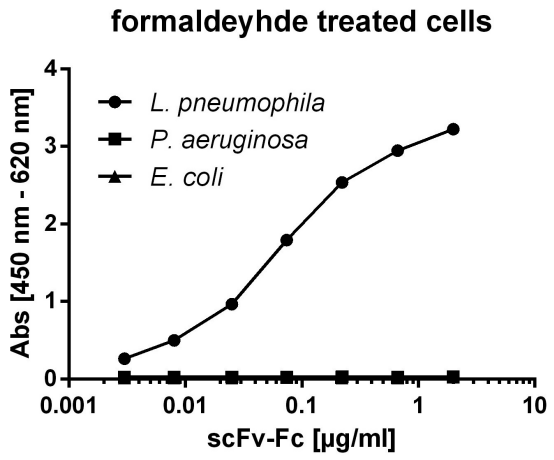

(c)

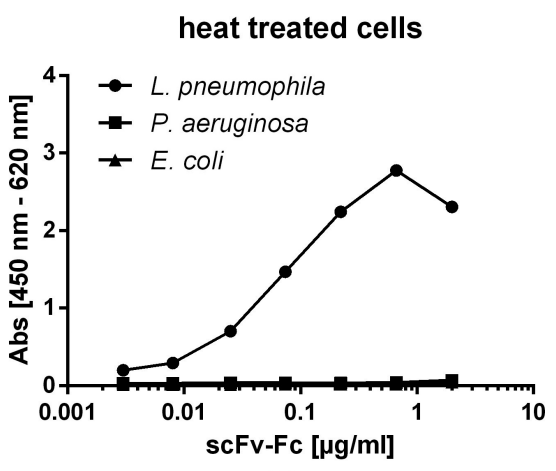

(b)

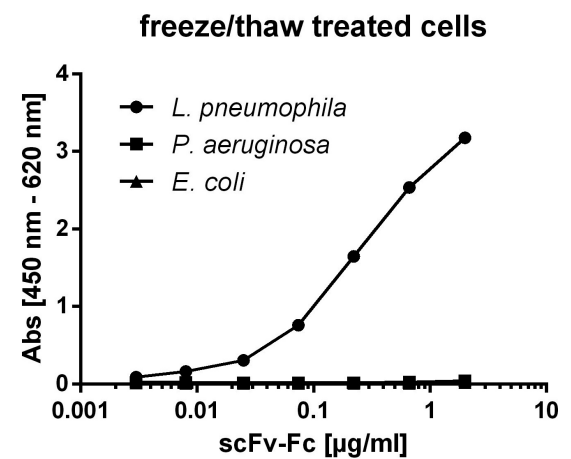

(d)

Fig. 2. Binding of PHK121-H2h to different cell preparations. Whole cell ELISA on L. pneumophila, P. aeruginosa and E. coli that were either (a) non-, (b) heat-, (c) formaldehyde- or (d) freeze/thaw treated. Binding of the scFv-Fc with human Fc part (PHK121-H2h) is shown.

dependent pannings of the human naïve scFv libraries HAL9/10 were performed on whole cells in suspension. To remove sticky and cross-reactive binders, the antibody phage libraries were first preincubated on BSA and formaldehyde-fixed $P$. aeruginosa. Nonbound antibody-phage were then selected on living L. pneumophila. After three panning rounds, around 600 clones were selected, used for the production of soluble monoclonal $\mathrm{scFv}$ antibody fragments and screened for specific cell binding on Legionella by ELISA (Fig. 1). In total, seven scFv clones were identified to bind specifically to Legionella but not to Pseudomonas cells with a signal to noise ratio of better than three. DNA sequence analysis of the isolated clones revealed that scFv PHK121-H2, was always selected in the independent pannings. This $\mathrm{scFv}$ clone also showed the strongest signal in ELISA and was chosen for further engineering. The monovalent $\mathrm{scFv}$ fragment PHK121-H2 was converted to a bivalent scFv-Fc fusion protein, carrying either a human (PHK121-H2h) or murine (PHK121-H2m) Fc part to facilitate its use in a sandwich-ELISA. Production of the scFv-Fc variants of PHK121-H2 in HEK293-6E cells, followed by Protein A affinity chromatography, resulted in yields of 105 and $122 \mathrm{mg}$ per L of culture volume and purities of $98 \%$ and $95 \%$ for the human Fc and mouse Fc versions, respectively.

Dilution series of PHK121-H2h and PHK121-H2m were tested in ELISA on whole cells of immobilized L. pneumophila, P. aeruginosa or E. coli cells that were either non-, heat-, formaldehyde- or freeze/thaw treated. Specific binding of the antibody was seen to all Legionella cell preparations but not to the other bacteria (Fig. 2). This was the first indication that the target on the cell surface may not be a protein. No significant differences were observed for the antibody versions carrying either human or murine Fc parts.

\subsection{Identification of the Legionella antigen bound by PHK121-H2h}

Since the panning was done on whole cells, the antigen recognized by $\mathrm{PHK} 121-\mathrm{H} 2 \mathrm{~h}$ was unknown. PHK121-H2h staining of western blots of total cell lysate of L. pneumophila showed multiple bands in the range of 25 to $65 \mathrm{kDa}$ that formed a ladder-like pattern described to be typical for LPS [25]. To confirm that the antigen is not a protein, the experiment 


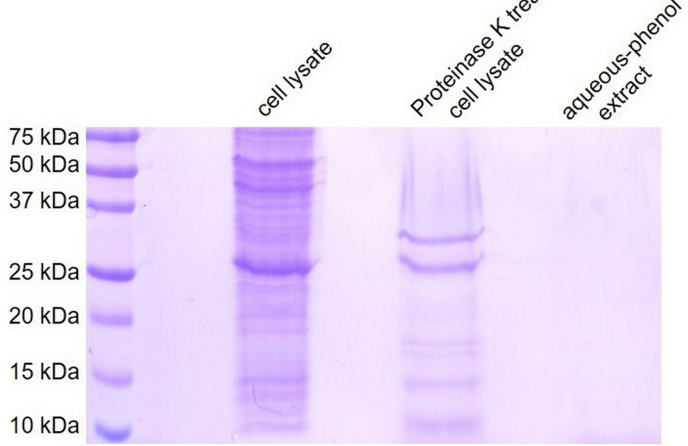

(a)

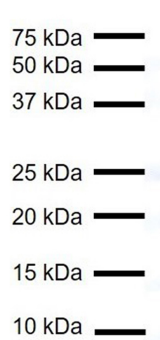

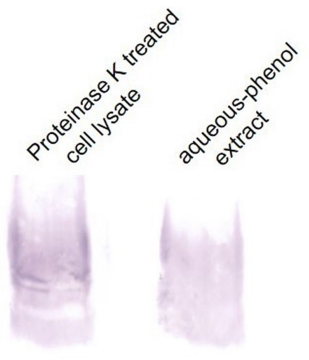

(b)

Fig. 3. Identification of the antigen bond by PHK121-H2h. SDS-PAGE analysis of whole cell lysate, Proteinase K-treated cell lysate and an aqueous-phenol extract of L. pneumophila by (a) Coomassie staining and (b) immunoblot staining with PHK121-H2h.

was repeated on Proteinase K-treated cell lysate. No difference in staining was observed, indicating binding to a non-protein target like LPS (Fig. 3). To further exclude residual proteins, a hot-phenol/water extraction of L. pneumophila cells was performed as described for the purification of LPS [12]. No proteins were detected in this preparation, yet reactivity with PHK121-H2h was still unchanged. The hypothesis that PHK121-H2h binds to LPS is also consistent with the resistance to chemical and physical denaturation conditions observed in ELISA before.

\subsection{Detection of L. pneumophila by sandwich-ELISA}

Direct ELISA is sensitive to impurities, which may hamper the analysis of diverse environmental specimens. Therefore, we developed a sandwich-ELISA with the aim to improve both specificity and sensitivity by specific capturing of cells. Here, L. pneumophila was first captured from a $2 \%$ BSA suspension with immobilized PHK121-H2m, carrying the murine Fc part. After that, the human Fc version PHK121$\mathrm{H} 2 \mathrm{~h}$ was used for detection of captured bacteria. Detection of L. pneumophila could be demonstrated down to a biomass that represents approximately 4,000 cells $(N=4, P<0.0001$, calculated by unpaired t test with Welch's correction) (Fig. 4).

\subsection{Specificity of PHK121-H2h}

So far, PHK121-H2h binding was only analyzed for the Corby strain of L. pneumophila. In order to evaluate the antibody's specificity in more detail, binding to a panel of six other Legionella strains and seven other Gram-negative bacteria that are commonly found in

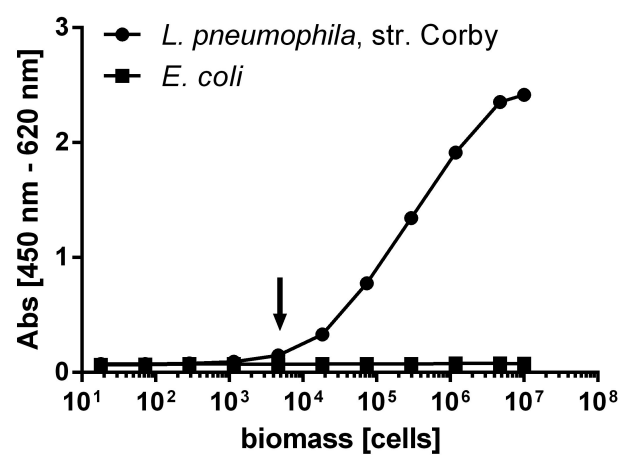

Fig. 4. Detection of L. pneumophila by sandwich-ELISA. Dilution series of heat treated L. pneumophila and E. coli cells were analyzed in a sandwich-ELISA, using the murine Fc version of PHK121-H2 for capture and its human Fc version for detection. Detection of $L$. pneumophila but not E. coli was observed. The black arrow indicates the detection limit that was determined to a biomass corresponding to 4000 cells $(N=4, P<0.0001)$.

water, was tested by immunoblot staining on cell lysate and by sandwich-ELISA on heat treated cells. Both methods revealed binding to the L. pneumophila strains Corby, Philadelphia-1 and Knoxville, which were all isolated from infected humans and belong to serogroup 1 (Fig. 5). Weak binding was also detected by ELISA but not by immunoblot to an environmental isolate of L. pneumophila that belonged to serogroup 1. No binding was observed to another environmental strain of serogroup 6 and to the species L. micdadei. Additionally, no binding to Citrobacter freundii, Escherichia coli, Klebsiella aerogenes, Pseudomonas aeruginosa, Raoultella planticola, Clostridium bifermentans and Clostridium perfringens was detected (Fig. 5).

Despite LPS was considered to be the antibody target, high specificity of the antibody was measured in particular for L. pneumophila from serogroup 1 which 


\section{L. pneumophila strains}

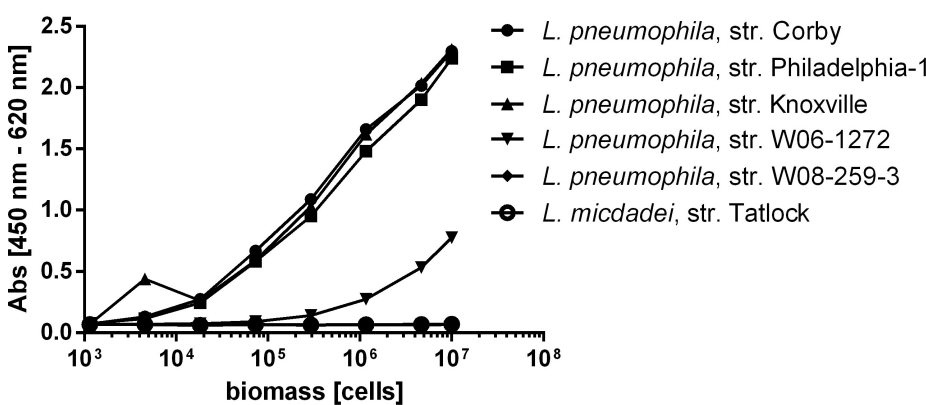

(a)
Gram-negative bacteria

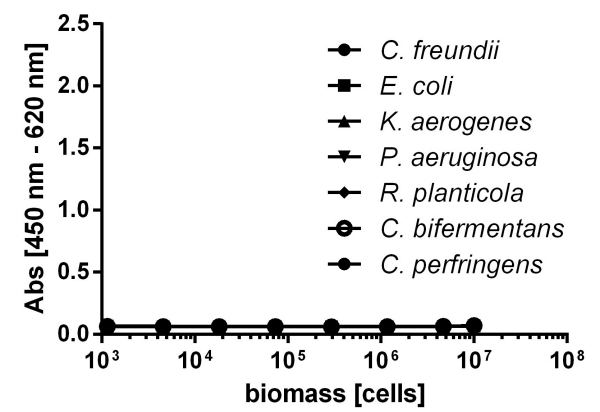

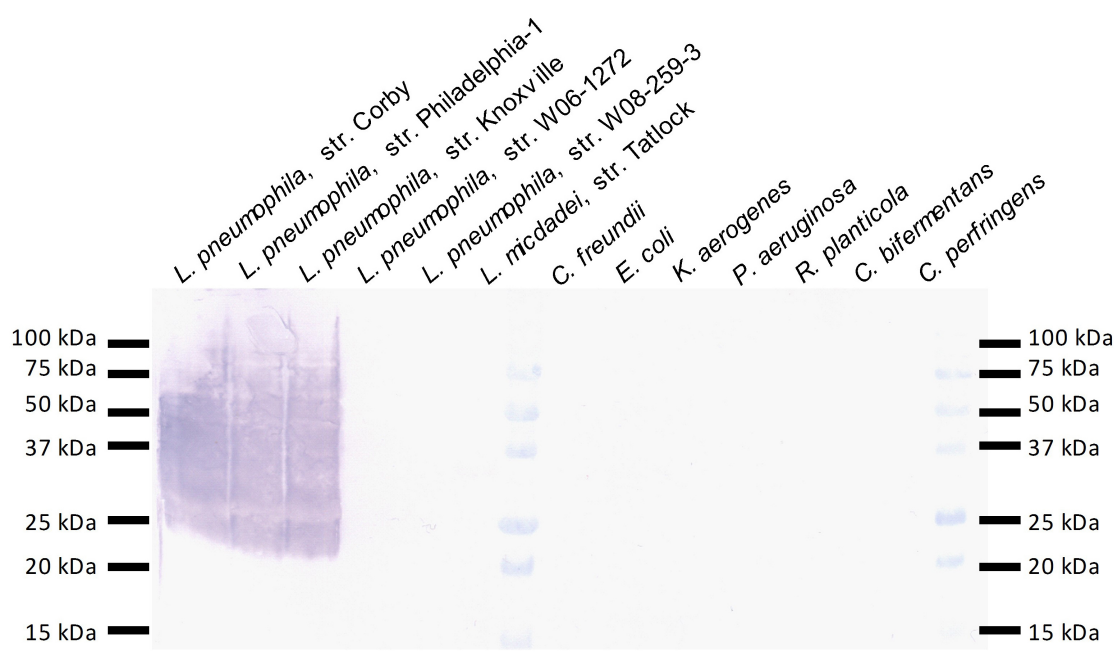

(b)

Fig. 5. Specificity of PHK121-H2h. Specificity of PHK121-H2h by (a) sandwich-ELISA and (b) immunoblot staining on different strains of $L$. pneumophila and various Gram-negative bacteria. ELISA was done as in Fig. 1; immunoblot was done as in Fig. 2.

were isolated from infected humans and thus expected to be very pathogenic.

\subsection{Detection of Legionella using an immunosensor}

To demonstrate that a fast and sensitive Legionella detection with PHK121-H2h is technically feasible on a portable device, an immunosensor was developed based on the electrochemical VR2 system from Vantix. First, stability of PHK121-H2h for long-term storage was assessed, a parameter important for the conceived use in point-of-care applications. For this purpose, the antibody was diluted to final assay concentration in $2 \%$ BSA and stored for up to 10 weeks at $4^{\circ} \mathrm{C}$, RT or $37^{\circ} \mathrm{C}$ before binding to Legionella was measured by ELISA. Storage for 10 weeks at $4{ }^{\circ} \mathrm{C}$ resulted in no reduction of the binding signal. $93 \%$ and $88 \%$ reactivity was re-

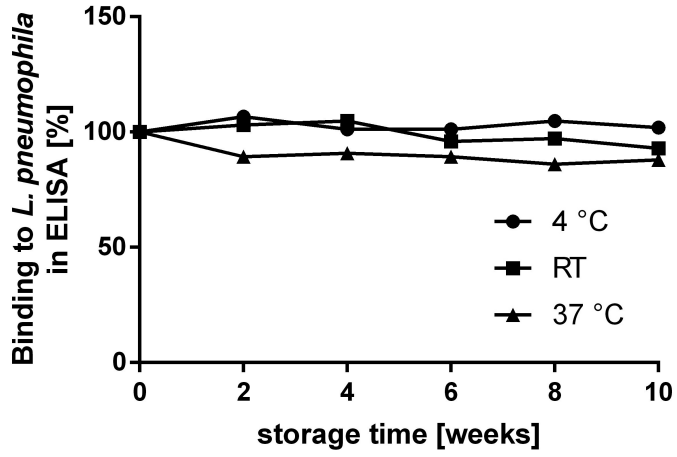

Fig. 6. Stability of PHK121-H2h. After dilution of the antibody to the final assay concentration, aliquots were stored for different time intervals at $4^{\circ} \mathrm{C}$, RT or $37^{\circ} \mathrm{C}$. Binding to L. pneumophila was measured by ELISA as described in Fig. 2.

tained after storage for 10 weeks at RT and $37^{\circ} \mathrm{C}$, respectively (Fig. 6). 


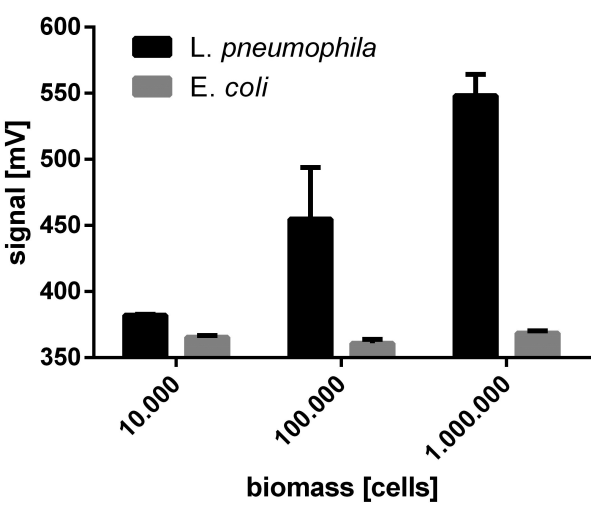

Fig. 7. Detection of L. pneumophila on the Vantix immunosensor system. Dilution series of heat treated L. pneumophila and E. coli were captured with PHK121-H2m and detected with PHK121-H2h using the Vantix VR2 system. Specific detection of L. pneumophila but no binding to $E$. coli was detected.

The Vantix biosensor assay was set up similar to the sandwich-ELISA. PHK121-H2m was immobilized and used for cell capture, while PHK121-H2h was used in combination with a secondary HRP conjugated antibody for detection. The catalytic activity of the enzyme created a shift in the electrochemical potential on the sensor surface that was recorded in real time. Analysis of the electric potential after 60 seconds revealed that a biomass corresponding to $10,000 \mathrm{Le}$ gionella cells could be easily distinguished from the background generated by E. coli (Fig. 7). The detection limit is comparable to the ELISA results, but was obtained within $35 \mathrm{~min}$ in a portable system.

\section{Discussion}

Since 2001, Legionella testing of drinking water is mandatory in Germany and regulated by the Trinkwasserverordnung (TrinkwV 2001). If more than $1 \mathrm{cfu} / \mathrm{ml}$ water is detected, local health authorities have to be informed and disinfection methods applied. Up to now, Legionella detection is done by the cultivation method which is regarded as gold standard. Yet, this requires several days to be conclusive. Faster alternatives were developed, including quantitative PCR [29], fluorescence in situ hybridization [8], flow cytometry [3] or ELISA [43]. In this work, we generated a human monoclonal recombinant antibody against $L$. pneumophila by antibody-phage display and applied it for specific detection of Legionella in a portable immunosensor.

So far, several reports demonstrated the successful use of antibody-phage display for the generation of diagnostic antibodies for the detection of pathogenic bacteria [31]. But to our knowledge, this is the first report of a successful selection of a recombinant human antibody against the pathogen $L$. pneumophila from a naïve human antibody library by antibody-phage display. The use of whole cells for panning resulted in a highly specific antibody which recognized a nonproteinaceous antigen which can be assumed to be cell wall lipopolysaccharides (LPS). LPS is of particular interest as a detection target, as it's abundance promises good sensitivity [38]. Second, it is highly stable [34], allowing detection even after a harsh sample treatment, which is helpful since water samples usually have to be concentrated significantly before detection. Third, it is the serogroup determining antigen of Legionella [10,39] that contains conserved as well as variable epitopes. That allows to do sero- and subtyping of Legionella as demonstrated previously [21]. The O-chain of Legionella LPS consists of a homopolymer of legionaminic acid [26] that exhibits distinct acetylation patterns. These are expected to mediate serologic specificity and antibody binding [28]. Since environmental samples of $L$. pneumophila serogroup 1 and 6 as well as $L$. micdadei were not detected sensitively, it might be possible that the antibody binds to a virulence marker of serogroup 1, as described previously with Mab3/1 [22]. In order to prove this hypothesis, a broader testing on other Legionella strains would be necessary. Significantly, no cross-reactivity was observed to seven other bacteria usually found in water samples, including members of Enterobacteriaceae, Pseudomonadaceae and Clostridiaceae. Using the sandwich-ELISA, a detection limit of 4,000 Legionella cells was reached, which would require a significant volume reduction of water samples to be of practical use. The necessary sample concentration units are under development, for example by using monolithic affinity filtration [41] or tangential flow filtration [9].

The novel human antibody to Legionella compares favorably to LPS specific Legionella antibodies generated by Hybridoma technology. For example, clone II-6-18 that is specific for L. pneumophila serogroup 1 showed a detection limit of 30,000 cells in ELISA [19, 42]. Clone 53-3D12 binds to a common epitope on LPS, allowing detection of L. pneumophila serogroup 1 to 8 [4]. Unfortunately, no reports were found where this antibody was used for quantitative detection of Legionella in a sandwich set-up, which might indicate that the antibody was not suited for this application. For example, stability and cross-reactivity issues are a problem often reported for Hybridoma de- 
rived antibodies (Bradbury et al., in preparation). Compared to that, our scFv-Fc antibody exhibited high stability, even when stored in finally diluted form at $37^{\circ} \mathrm{C}$. A very similar approach to our work using antibody phage-display was done by Griep et al. who used purified LPS of Ralstonia solanacearum Race 3 for antibody selection [18]. The selected antibodies were used in combination with a polyclonal capture antisera in order to detect bacterial cells by sandwich-ELISA. High sensitivity of $5 \times 10^{3} \mathrm{cfu} / \mathrm{ml}$ was reached, but a polyclonal antiserum was still needed, adding the disadvantages of limited resource and complex undefined composition. Furthermore, this LPS antibody crossreacted with other organisms, rendering very much its relevance for diagnostics [18].

Another antibody-based sensor system was recently described by Wunderlich [48], who used monolithic adsorption filtration (MAF) with centrifugal ultrafiltration (CeUF) to concentrate $10 \mathrm{~L}$ of water to $1 \mathrm{~mL}$. The concentrated sample was applied by microfluidics on a microarray (CL-SMIA), where the Legionella cells were captured by polyclonal or monoclonal LPS antibodies. The detection was performed with a biotinylated polyclonal antibody in combination with a HRP conjugate. Based on the number of bound cells, a chemiluminescent signal was generated and optically analyzed. Using this system, a detection limit of approximately 1,500 cells was reached. Regarding that these cells were present in $10 \mathrm{~L}$ water, a sensitivity of $0.39 \mathrm{cfu} / \mathrm{mL}$ was achieved [48]. This is the first indication that a sensitivity of $1 \mathrm{cfu} / \mathrm{mL}$ can be reached which is required for Legionella diagnostic by law. Combinations of improved water concentration and biosensors may provide a practical solution in the future. Also, the affinity of recombinant antibodies can be quite reliably improved by in vitro evolution [27]. Furthermore, our antibody shows specificity only to Legionella pneumophila of serogroup 1. This group contains with $84 \%$ of all infections the most important pathogenic Legionella [49]. Yet, since all Legionella species are regarded as potential pathogenic [40], a diagnostic test requires broad specificity to different $L e$ gionella serogroups and species. Thus, the specificity of a point of care test device needs to be extended to other subtypes, serogroups or species. As demonstrated recently, a single amino acid exchange in the CDR region can be sufficient to alter the antibody's specificity to Legionella serotypes [36]. This may allow specificity expansion of PHK121-H2 by random mutagenesis and re-selection by phage display.

To our knowledge, this is the first report that demonstrated successful generation of a potentiometric im- munosensor for the detection of L. pneumophila. Using this portable technology, the assay time could be reduced to $35 \mathrm{~min}$ with a detection level still comparable to the tedious sandwich-ELISA.

\section{Acknowledgments}

This project was funded by Eurostars Project E! 6994. We would like to thank Doris Meier and Saskia Helmsing for excellent technical support.

\section{References}

[1] Y.A. Kwaik, L.Y. Gao, B.J. Stone, C. Venkataraman and O.S. Harb, Invasion of protozoa by Legionella pneumophila and its role in bacterial ecology and pathogenesis, Appl Environ Microbiol 64 (1998), 3127-3133.

[2] D.G. Addiss, J.P. Davis, M. LaVenture, P.J. Wand, M.A. Hutchinson and R.M. McKinney, Community-acquired Legionnaires' disease associated with a cooling tower: Evidence for longer-distance transport of Legionella pneumophila, Am J Epidemiol 130 (1989), 557-568.

[3] H. Aurell, P. Catala, P. Farge, F. Wallet, M. Le Brun, J.H. Helbig, S. Jarraud and P. Lebaron, Rapid detection and enumeration of Legionella pneumophila in hot water systems by solidphase cytometry, Appl Environ Microbiol 70 (2004), 16511657.

[4] C. Barthe, J.R. Joly, D. Ramsay, M. Boissinot and N. Benhamou, Common epitope on the lipopolysaccharide of Legionella pneumophila recognized by a monoclonal antibody, J Clin Microbiol 26 (1988), 1016-1023.

[5] A. Borges, M. Simões, A. Martínez-Murcia and M. Saavedra, Detection of Legionella SPP in natural and man-made water systems using standard guidelines, Journal of Microbiology Research 2 (2012), 95-102.

[6] J.A. Bozue and W. Johnson, Interaction of Legionella pneumophila with Acanthamoeba castellanii: Uptake by coiling phagocytosis and inhibition of phagosome-lysosome fusion, Infect Immun 64 (1996), 668-673.

[7] D.J. Brenner, A.G. Steigerwalt and J.E. McDade, Classification of the Legionnaires' disease bacterium: Legionella pneumophila, genus novum, species nova, of the family Legionellaceae, familia nova, Ann Intern Med 90 (1979), 656-658.

[8] S. Buchbinder, K. Trebesius and J. Heesemann, Evaluation of detection of Legionella SPP in water samples by fluorescence in situ hybridization, PCR amplification and bacterial culture, Int J Med Microbiol 292 (2002), 241-245.

[9] L. Cai, Y. Yang, N. Jiao and R. Zhang, Evaluation of tangential flow filtration for the concentration and separation of bacteria and viruses in contrasting marine environments, PLoS One 10 (2015), e0136741.

[10] C.A. Ciesielski, M.J. Blaser and W.L. Wang, Serogroup specificity of Legionella pneumophila is related to lipopolysaccharide characteristics, Infect Immun 51 (1986), 397-404.

[11] N. Coetzee, H. Duggal, J. Hawker, S. Ibbotson, T.G. Harrison, N. Phin, V. Laza-Stanca, R. Johnston, Z. Iqbal, Y. Rehman, E. Knapper, S. Robinson and N. Aigbogun, An outbreak of Legionnaires' disease associated with a display spa pool in retail premises, Stoke-on-Trent, United Kingdom, July 2012, Euro Surveill 17 (2012). 
[12] M.R. Davis, Jr. and J.B. Goldberg, Purification and visualization of lipopolysaccharide from Gram-negative bacteria by hot aqueous-phenol extraction, J Vis Exp (2012).

[13] R.B. Fitzgeorge, A. Baskerville, M. Broster, P. Hambleton and P.J. Dennis, Aerosol infection of animals with strains of Legionella pneumophila of different virulence: Comparison with intraperitoneal and intranasal routes of infection, $J$ Hyg (Lond) 90 (1983), 81-89.

[14] C.B. Fliermans, W.B. Cherry, L.H. Orrison, S.J. Smith, D.L Tison and D.H. Pope, Ecological distribution of Legionella pneumophila, Appl Environ Microbiol 41 (1981), 9-16.

[15] D.W. Fraser, T.R. Tsai, W. Orenstein, W.E. Parkin, H.J. Beecham, R.G. Sharrar, J. Harris, G.F. Mallison, S.M. Martin, J.E. McDade, C.C. Shepard and P.S. Brachman, Legionnaires' disease: Description of an epidemic of pneumonia, $N$ Engl J Med 297 (1977), 1189-1197.

[16] A. Frenzel, J. Kügler, S. Wilke, T. Schirrmann and M. Hust, Construction of human antibody gene libraries and selection of antibodies by phage display, Methods Mol Biol 1060 (2014), 215-243.

[17] L.Y. Gao, O.S. Harb and Y.A. Kwaik, Utilization of similar mechanisms by Legionella pneumophila to parasitize two evolutionarily distant host cells, mammalian macrophages and protozoa, Infect Immun 65 (1997), 4738-4746.

[18] R.A. Griep, C. van Twisk, J.R. van Beckhoven, J.M. van der Wolf and A. Schots, Development of specific recombinant monoclonal antibodies against the lipopolysaccharide of ralstonia solanacearum race 3, Phytopathology 88 (1998), 795803.

[19] J.G. Guillet, J. Hoebeke, C. Tram, S. Marullo and A.D. Strosberg, Characterization, serological specificity, and diagnostic possibilities of monoclonal antibodies against Legionella pneumophila, J Clin Microbiol 18 (1983), 793-797.

[20] D. Harper, Minimising the spread of Legionnaires' disease a programme designed to prevent incubation of LP in hospital water and air conditioning systems, Hosp Eng 38 (1984), 5-9.

[21] J.H. Helbig, J.B. Kurtz, M.C. Pastoris, C. Pelaz and P.C Luck, Antigenic lipopolysaccharide components of Legionella pneumophila recognized by monoclonal antibodies: Possibilities and limitations for division of the species into serogroups, J Clin Microbiol 35 (1997), 2841-2845.

[22] J.H. Helbig, P.C. Luck, Y.A. Knirel, W. Witzleb and U. Zahringer, Molecular characterization of a virulenceassociated epitope on the lipopolysaccharide of Legionella pneumophila serogroup 1, Epidemiol Infect 115 (1995), 7178.

[23] V. Jager, K. Bussow, A. Wagner, S. Weber, M. Hust, A. Frenzel and T. Schirrmann, High level transient production of recombinant antibodies and antibody fusion proteins in HEK293 cells, BMC Biotechnol 13 (2013), 52.

[24] R.I. Jepras, R.B. Fitzgeorge and A. Baskerville, A comparison of virulence of two strains of Legionella pneumophila based on experimental aerosol infection of guinea-pigs, J Hyg (Lond) 95 (1985), 29-38.

[25] D. Jurgens and F.J. Fehrenbach, Cross-reacting lipopolysaccharide antigens in Legionella pneumophila serogroups 1 to 14, Infect Immun 63 (1995), 2180-2184.

[26] Y.A. Knirel, E.T. Rietschel, R. Marre and U. Zahringer, The structure of the O-specific chain of Legionella pneumophila serogroup 1 lipopolysaccharide, Eur J Biochem 221 (1994), 239-245.

[27] N. Kobayashi, H. Oyama, Y. Kato, J. Goto, E. Soderlind and C.A. Borrebaeck, Two-step in vitro antibody affinity maturation enables estradiol-17beta assays with more than 10-fold higher sensitivity, Anal Chem 82 (2010), 1027-1038.

[28] O. Kooistra, E. Luneberg, B. Lindner, Y.A. Knirel, M. Frosch and U. Zahringer, Complex O-acetylation in Legionella pneumophila serogroup 1 lipopolysaccharide, evidence for two genes involved in 8-O-acetylation of legionaminic acid, Biochemistry 40 (2001), 7630-7640.

[29] L.H. Krojgaard, K.A. Krogfelt, H.J. Albrechtsen and S.A Uldum, Detection of Legionella by quantitative-polymerase chain reaction (qPCR) for monitoring and risk assessment, BMC Microbiol 11 (2011), 254

[30] J. Kügler, S. Wilke, D. Meier, F. Tomszak, A. Frenzel, T. Schirrmann, S. Dubel, H. Garritsen, B. Hock, L. Toleikis, M. Schutte and M. Hust, Generation and analysis of the improved human HAL9/10 antibody phage display libraries, BMC Biotechnol 15 (2015), 10.

[31] P. Kuhn, V. Fuhner, T. Unkauf, G.M. Moreira, A. Frenzel, S Miethe and M. Hust, Recombinant antibodies for diagnostics and therapy against pathogens and toxins generated by phage display, Proteomics Clin Appl (2016).

[32] X. Li, D. Lu, Z. Sheng, K. Chen, X. Guo, M. Jin and H. Han, A fast and sensitive immunoassay of avian influenza virus based on label-free quantum dot probe and lateral flow test strip, Talanta 100 (2012), 1-6.

[33] C.E. Lucas, T.H. Taylor, Jr. and B.S. Fields, Accuracy and precision of Legionella isolation by US laboratories in the ELITE program pilot study, Water Res 45 (2011), 4428-4436.

[34] P.O. Magalhaes, A.M. Lopes, P.G. Mazzola, C. Rangel-Yagui, T.C. Penna and A. Pessoa, Jr., Methods of endotoxin removal from biological preparations: a review, J Pharm Pharm Sci 10 (2007), 388-404.

[35] E. Mauriz, A. Calle, J.J. Manclus, A. Montoya, A. Hildebrandt, D. Barcelo and L.M. Lechuga, Optical immunosensor for fast and sensitive detection of DDT and related compounds in river water samples, Biosens Bioelectron 22 (2007), 1410-1418.

[36] B.J. McCarthy and A.S. Hill, Altering the fine specificity of an anti-Legionella single chain antibody by a single amino acid insertion, J Immunol Methods 251 (2001), 137-149.

[37] G.K. Morris, C.M. Patton, J.C. Feeley, S.E. Johnson, G. Gorman, W.T. Martin, P. Skaliy, G.F. Mallison, B.D. Politi and D.C. Mackel, Isolation of the Legionnaires' disease bacterium from environmental samples, Ann Intern Med 90 (1979), 664 666.

[38] H. Nikaido, Outer membrane, Escherichia Coli and Salmonella (1996), 29-47.

[39] F.S. Nolte, C.A. Conlin and M.A. Motley, Electrophoretic and serological characterization of the lipopolysaccharides of Legionella pneumophila, Infect Immun 52 (1986), 676-681.

[40] M. Palusinska-Szysz and M. Cendrowska-Pinkosz, Pathogenicity of the family Legionellaceae, Arch Immunol Ther Exp (Warsz) 57 (2009), 279-290.

[41] L. Pei, M. Rieger, S. Lengger, S. Ott, C. Zawadsky, N.M. Hartmann, H.C. Selinka, A. Tiehm, R. Niessner and M. Seidel, Combination of crossflow ultrafiltration, monolithic affinity filtration, and quantitative reverse transcriptase PCR for rapid concentration and quantification of model viruses in water, Environ Sci Technol 46 (2012), 10073-10080.

[42] F. Petitjean, J.G. Guillet, B. Vray, J. Hoebeke, C. Tram and A.D. Strosberg, Standardization for diagnostic purposes of a monoclonal antibody against Legionella pneumophila, Dev Biol Stand 57 (1984), 99-105.

[43] U. Reidt, B. Geisberger, C. Heller and A. Friedberger, Automated immunomagnetic processing and separation of Legionella pneumophila with manual detection by sandwich 
ELISA and PCR amplification of the ompS gene, J Lab Autom 16 (2011), 157-164.

[44] Robert-Koch-Institut, Legionãrskrankheit in Deutschland (2001 bis 2013), in: Epidemiologisches Bulletin, Nr 13, 2015.

[45] T.J. Rowbotham, Preliminary report on the pathogenicity of Legionella pneumophila for freshwater and soil amoebae, $J$ Clin Pathol 33 (1980), 1179-1183.

[46] J.O. Tobin, J. Beare, M.S. Dunnill, S. Fisher-Hoch, M. French, R.G. Mitchell, P.J. Morris and M.F. Muers, Legionnaires' disease in a transplant unit: Isolation of the causative agent from shower baths, Lancet 2 (1980), 118-121.

[47] H. von Baum, S. Ewig, R. Marre, N. Suttorp, S. Gonschior, T. Welte, C. Luck and G. Competence, Network for community acquired pneumonia study, community-acquired le- gionella pneumonia: New insights from the German competence network for community acquired pneumonia, Clin Infect Dis 46 (2008), 1356-1364.

[48] A. Wunderlich, C. Torggler, D. Elsasser, C. Luck, R. Niessner and M. Seidel, Rapid quantification method for Legionella pneumophila in surface water, Anal Bioanal Chem 408 (2016), 2203-2213

[49] V.L. Yu, J.F. Plouffe, M.C. Pastoris, J.E. Stout, M. Schousboe, A. Widmer, J. Summersgill, T. File, C.M. Heath, D.L. Paterson and A. Chereshsky, Distribution of Legionella species and serogroups isolated by culture in patients with sporadic community-acquired legionellosis: An international collaborative survey, J Infect Dis 186 (2002), 127-128. 